ACCEPTED MANUSCRIPT

\title{
Novel Three-Dimensional Interphase Characterisation of Polymer Nanocomposites Using Nanoscaled Topography
}

To cite this article before publication: Mohanad Mousa et al 2018 Nanotechnology in press https://doi.org/10.1088/1361-6528/aacd5d

\section{Manuscript version: Accepted Manuscript}

Accepted Manuscript is "the version of the article accepted for publication including all changes made as a result of the peer review process, and which may also include the addition to the article by IOP Publishing of a header, an article ID, a cover sheet and/or an 'Accepted Manuscript' watermark, but excluding any other editing, typesetting or other changes made by IOP Publishing and/or its licensors"

This Accepted Manuscript is @ 2018 IOP Publishing Ltd.

During the embargo period (the 12 month period from the publication of the Version of Record of this article), the Accepted Manuscript is fully protected by copyright and cannot be reused or reposted elsewhere.

As the Version of Record of this article is going to be / has been published on a subscription basis, this Accepted Manuscript is available for reuse under a CC BY-NC-ND 3.0 licence after the 12 month embargo period.

After the embargo period, everyone is permitted to use copy and redistribute this article for non-commercial purposes only, provided that they adhere to all the terms of the licence https://creativecommons.org/licences/by-nc-nd/3.0

Although reasonable endeavours have been taken to obtain all necessary permissions from third parties to include their copyrighted content within this article, their full citation and copyright line may not be present in this Accepted Manuscript version. Before using any content from this article, please refer to the Version of Record on IOPscience once published for full citation and copyright details, as permissions will likely be required. All third party content is fully copyright protected, unless specifically stated otherwise in the figure caption in the Version of Record.

View the article online for updates and enhancements. 
6 Abstract

\section{Novel Three-Dimensional Interphase Characterisation of Polymer}

\section{Nanocomposites Using Nanoscaled Topography}

Mohanad Mousa and Yu Dong*,

School of Civil and Mechanical Engineering, Curtin University, GPO Box U1987, Perth, WA

\section{5, Australia}

Mechanical properties of polymer nanocomposites depend primarily on nanointerphases as

transitional zones between nanoparticles and surrounding matrices. Due to the difficulty in the quantitative characterisation of nanointerphases, previous literatures generally deemed such interphases as one-dimensional uniform zones around nanoparticles by assumption for analytical or theoretical modelling. We hereby have demonstrated for the first time direct three-dimensional topography and physical measurement of nanophase mechanical properties between nanodimeter bamboo charcoals (NBCs) and poly (vinyl alcohol) (PVA) in polymer nanocomposites. Topographical features, nanomechanical properties and dimensions of nanointerphases were systematically determined via peak force quantitative nanomechanical tapping mode (PFQNM). Significantly different mechanical properties of nanointerphases were revealed as opposed to those of individual NBCs and PVA matrices. Non-uniform irregular three-dimensional structures and shapes of nanointerphases are manifested around individual NBCs, which can be greatly influenced by nanoparticle size and roughness, and nanoparticle dispersion and distribution. Elastic moduli of nanointerphases were experimentally determined in range from $25.32 \pm 3.4$ to $66.3 \pm 3.2 \mathrm{GPa}$. Additionally, it is clearly shown that the interphase modulus strongly depends on interphase surface area $S A_{\text {Interphase }}$ and interphase volume $V_{\text {Interphase }}$ Different NBC distribution patterns from fully to

* Corresponding author. Tel.: +61 89266 9055; fax: +61 892662681.

E-mail address: Y.Dong@curtin.edu.au (Y. Dong). 
1 partially embedded nanoparticles are proven to yield a remarkable reduction in elastic moduli

2 of nanointerphases.

3 Keywords: polymer nanocomposites; polyvinyl alcohol (PVA); nanodiameter bamboo

$4 \quad$ charcoals (NBCs); interphases; nanomechanical properties

\section{1. Introduction}

6 In a nanocomposite system, mechanical reinforcement is based on a fundamental concept that

7 the chain mobility of soft matrices is constrained by much stiffer nanoparticles [1]. As a

8 consequence, an effective load transfer occurs from matrices to fillers such as nanoparticles

9 to carry a disproportionally high fraction of applied loads, thus leading to an increase in load

10 resistance [1]. This phenomenon is well known to be associated with the level of interfacial

11 bonding between nanoparticles and polymer matrices in terms of interphase existence,

12 dimensions, structures and compositions [2]. Hence, crack initiation or propagation may take

13 place in a nanocomposite system, resulting from the lack of effective load transfer owing to

14 the weak interfacial bonding.

Interfaces are described as a material boundary between two or more phases with distinct

16 chemical/physical properties and morphological structures. Furthermore, a material volume

17 influenced by the interfacial interaction can be named 'interphase' [3, 4]. Interphase regions

18 start from the interfacial boundary of nanofillers with different properties from those of bulk

19 nanofillers and end where they are in connection with polymer matrices whose properties

20 also vary from bulk matrices [4-6]. The material performance of nanocomposites is primarily

21 impacted by the interphase regions where structural and chemical changes such as

22 crosslinking density and crystalline phases result in a major alteration to composite bulk

23 properties [3]. It is also worth mentioning that the alteration in the mobility of polymeric

24 chains plays an important role in mechanical and dielectric properties of nanocomposites [7]. 
1 Due to large interfacial area to volume ratios in nanocomposites, interfacial regions consist of

2 a significant portion of bulk nanocomposites. For instance, with the addition of 5 vol\%

3 monodispersed spherical nanoparticles (particle diameter of $10 \mathrm{~nm}$ and interphase thickness

4 of $0.5 \mathrm{~nm}$ ), the volume fraction of interphases can be as high as 25 vol\% [7]. More

5 impressively, when particle diameter is reduced to less than $5 \mathrm{~nm}$, the volume fraction of

6 interphases is increased by over $50 \mathrm{vol} \%$ as compared to that of particles [7]. The

7 characterisation of existing interphases and their associated properties is often difficult to

8 undertake as interphases for nanocomposites are generally on a nanoscaled level, and thus

9 researchers have had to make most experimental efforts in an uncrosslinked state as the

10 indirect evidence [8-10]. For example, chain mobility near interphase regions can be less than

11 those of polymer matrices in a nanocomposite system [9, 10]. As a result, Litvinov and

12 Steeman [11] employed proton, low resolution $T_{2}$ nuclear magnetic resonance (NMR)

13 relaxation technique to detect existing interphases between ethylene propylene dine

14 monomers (EPDMs) and carbon blacks. It is indicative of a significant difference in the chain

15 mobility of EPDMs near carbon black surfaces at which the generated layer sizes of 16 immobilised EPDMs were estimated in range of 1-2 for the unit diameter of monomers [11].

17 Pompe and Mäder [12] identified interphases according to differential scanning calorimetry

(DSC) in polypropylene/glass fibre composites, which, however, was limited to 19 semicrystalline polymers as matrices in composite materials with only high glass fibre 20 contents. Brown et al. [13] studied a relationship between nanoparticle diameter and 21 interphase thickness based on molecular dynamic (MD) simulations, which implied that the interphase thickness appeared to be relatively insensitive to nanoparticle diameters and contents. Li et al. [14] reported that the volume fraction of interphases could be size-

24 independent by using a modified hierarchical multi-interphase model (MHMM). However, 25 the interphase thickness might be influenced by the reinforcing efficiency of nanoparticles 
1 when their lengths were over $40 \mathrm{~nm}$. On the contrary, Gu et al. [15] and other co-workers

$2[16,17]$ inferred that the interphase thickness could be non-constant in nanocomposites

3 systems. So far, nanointerphase properties and features have not been explicitly quantified in

4 a systematic manner by means of direct topography of nanomechanical characterisation. As a

5 matter of fact, nanomechanical techniques using tip-sample interactions such as atomic force

6 microscopy (AFM) [18, 19], nanoindentation and nanoscratch tests [20-22] are vital as

7 effective and relatively straightforward approaches to determine nanomechanical properties

8 of interphases. In addition, interphase dimensions and sizes can be clearly specified due to

9 their distinct mechanical properties from those of bulk materials. The interphase width of

10 glass fibres coated with coupling agents was previously revealed to be around several

11 microns $[8,20,23]$. Nonetheless, a quantitative analysis of interphase dimensions associated

12 with nanomechanical properties still undergoes limited lateral resolutions and positioning capability of indenter probe used in nanoindentation, and nanoscratch techniques. In particular, interphase regions of thermosetting polymer/glass fibre composites are much thinner than those of individual fibre and matrix components [22, 24, 25]. Plastic deformation usually takes place under a high fraction of applied load, thus resulting in an increase in the minimum allowable distance between two indentation spots, as well as the reduction of lateral resolutions of such tests $[8,24,25]$. The peak force quantitative nanomechanical mapping (PFQNM) becomes a relatively new and powerful technique to quantitatively measure nanomechanical properties of materials such as stiffness and adhesion of nanocomposites along with corresponding acquired dimensions [26-30]. The use of PFQNM greatly supports the measurement of material elastic properties based on tip-sample force curves and acquisition of topographic images simultaneously. Moreover, other critical properties, consisting of tip-surface adhesion and surface deformation, can also be obtained 25 by avoiding the difficulty associated with lateral forces. Such a technique is believed not only 
1 to sophisticatedly distinguish between nanofillers, nanointerphases and polymer matrices, but

2 also to accurately quantify dimensions and nanomechanical properties of interphases.

The increasing interest in bionanocomposites becomes more pronounced after great concerns of environmental issues are raised associated with the applications and manufacture 5 of conventionally synthetic nanocomposites [1, 31]. In particular, PVA is a typical water6 soluble biopolymer with excellent flexibility [32], biocompatibility and biodegradability [32,

7 33] as well as recyclability and biotribological properties [34]. On the other hand, NBCs are

8 effective carbon-based nanofillers due to their extraordinary material features such as high

from those of their bulk material counterparts. It is worth noting that average elastic modulus of bulk PVA films is approximately $2.08 \mathrm{GPa}$ at a macroscopic level [36], which is far smaller in magnitude when compared with that of local nanophases for PVA blended with 10 wt\% poly(acrylic acid) (PAA) at $9.9 \mathrm{GPa}$ [37]. Moreovere, in case of PVA-PAA based nanocomposites reinforced with cellulose $10 \mathrm{wt} \%$ nanocrystals (CNCs) [37], their average elastic modulus in the interphase was found to vary from $12.8 \mathrm{GPa}$ at the $\mathrm{CNC}$ interface to 9.9 GPa in PVA-PPA matrices. On the contrary, PVA nanocomposites reinforced with 10 wt $\%$ CNCs demonstrated the highest elastic modulus of only $1.9 \mathrm{GPa}$ in their bulk properties [38]. This modulus variation phenomenon between nanomechanical properties and bulk properties is not limited to PVA alone, but can be applied to different polymers or composites. For instance, the storage modulus of epoxy was measured to be $17 \mathrm{GPa}$ via nanomechanical measurement as opposed to 3-4 GPa for their bulk composites obtained by tensile tests [15]. Moreover, elastic modulus of bulk poly (ether sulfone) (PESU) membrane 
1 substrates was detected to be only $151.7 \pm 7.9 \mathrm{MPa}$ [39] when compared to $3.2 \pm 0.3 \mathrm{GPa}$ for 2 PESU films at a nanoscaled level [40].

3

4

As mentioned earlier, the major drawback in previous literatures depended on the assumption of the interphase as homogeneous layers formed around nanoparticles with uniform thickness. The utilisation of such a simplified concept in theoretical modeling according to Mori-Tanaka model [41] and Maxwell model [42] has led to little success in order to predict mechanical properties of nanocomposites. In this study, 3D nanointerphase topography and characterisation have been demonstrated for the first time when embedded with anisotropic NBCs in PVA/NBC nanocomposites. The actual measurement of nanointerphase dimensions and properties is pivotal to evaluate the interaction level between NBCs and PVA matrices in such nanocomposites. Relevant results are particularly important for better estimation of nanocomposite properties based on real nanointerphase behavior in terms of volume fraction and surface area [43].

\section{Materials and methods}

\subsection{Materials and film fabrication}

PVA biopolymer (material type: MFCD00081922) with average molecular weight $M_{w}=89000-98000 \mathrm{~g} / \mathrm{mol}$ and a degree of hydrolysis over $99 \%$ was purchased from Sigma Aldrich Pty. Ltd, Australia. Highly bioactive NBC particles (particle diameter: <100 nm) in disk-like shape was supplied by US Research Nanomaterials, Inc. Co., USA with the surface area of $624.81 \mathrm{~m}^{2} / \mathrm{g}^{-1}$ and pore area of $415 \mathrm{~m}^{2} / \mathrm{g}^{-1}[36]$.

PVA/NBC nanocomposite films were fabricated by simple solution casting. Initially, 5 wt\%/v PVA aqueous solution was prepared by mixing $10 \mathrm{~g}$ PVA with $190 \mathrm{ml}$ deionised water using a vigorous magnetic stirrer at $400 \mathrm{rpm}$ and $90^{\circ} \mathrm{C}$ for $3 \mathrm{~h}$ until PVA was completely dissolved to prepare the stock solution. Aqueous suspensions of NBCs were obtained using mechanical mixing in deionised water with a rotor speed of $405 \mathrm{rpm}$ at $40^{\circ} \mathrm{C}$ 
1 for $2 \mathrm{~h}$. Afterwards such a mixture underwent the ultrasonication (Model ELMA Ti-H-5) at

$225 \mathrm{kHz}$ and $40^{\circ} \mathrm{C}$ with a power intensity of $70 \%$ for $1 \mathrm{~h}$ in order to break up aggregated

3 particles and evenly disperse NBCs in deionised water. NBC loadings of $0,3,5$ and 10 wt $\%$

4 were then achieved by adding appropriate amounts of PVA. NBC aqueous suspensions were

5 gradually added in a dropwise manner into PVA solutions, simultaneously/subjected to

6 mechanical mixing at $405 \mathrm{rpm}$ and $40^{\circ} \mathrm{C}$ for $2 \mathrm{~h}$. Then their mixtures were stirred at $400 \mathrm{rpm}$

7 and $90^{\circ} \mathrm{C}$ for $1 \mathrm{~h}$ prior to subsequent sonication for $30 \mathrm{~min}$ to achieve uniform NBC

8 dispersion. Finally prepared $20 \mathrm{ml}$ solution was cast on a glass petri dish, and was solidified

9 and dried in an air-circulating oven at $40^{\circ} \mathrm{C}$ for $48 \mathrm{~h}$. Finally, prepared films were peeled off

10 and transferred to a silica gel-contained desiccator for material storage before any further

11 testing and analysis.

\subsection{Characterisation method}

Nanomechanical properties of PVA/NBC nanocomposites were quantatively assessed via atomic force microscopy (AFM) in a peak force quantitative nanomechanical tapping mode (PFQNM) for this study $[44,45]$. This new technique enables to measure nanomechanical properties such as elastic modulus and adhesion force of nanocomposite films (see supporting information for more details) [45-48].

In this study, a Bruker Dimension Fastscan AFM system was employed to measure peak force quantitative nanomechanical properties and acquire single force-distance curve under the ambient condition. Moreover, RTESPA 525A probes, with a nominal spring constant of $200 \mathrm{~N} / \mathrm{m}$, a nominal tip radius of $8 \mathrm{~nm}$ and a nominal resonant frequency of $525 \mathrm{kHz}$, were utilised for the direct measurement of correponding nanomechanical properties. Before each measurment, it was confirmed that the deflection sensitivity was calibrated by obtaining a 24 force curve based on the stiff sapphire-12 surface. Later on, a thermal tuning method [44] 25 was employed to determine the spring constant (See supporting information for more details) 
1 [49-51]. AFM imaging analysis was undertaken with the TESPA probe at the nominal spring

2 constant of $40 \mathrm{~N} / \mathrm{m}$ and tip radius of $8 \mathrm{~nm}$. The image scan rate was kept at $2 \mathrm{~Hz}$ with 256

$3 \times 256$ digital pixel resolution. AFM topographic images were first-order flattened via Flatten

4 command in Burker Nanoscope 1.5 software, which was used to remove unwanted features

5 resulting from the vertical $(\mathrm{Z})$ scanner such as noise, bow and tilt.

\section{2.3. Modeling approach}

\section{$7 \quad 2.3 .1$ Interphase modulus}

8 The average elastic properties of interphases between PVA matrices and dispersed 9 anisotropic NBCs in various shapes and sizes were determined according to a data set of 10 elastic moduli collected based on PVA-NBC interphases surrounding 75 different NBCs at 25 11 line scan regions (LSRs).

\subsubsection{Interphase dimension}

13 Interphase dimensions in terms of interphase width $W_{\text {Interphase, }}$ interphase length $L_{\text {Interphase }}$ and 14 interphase height $H_{\text {Interphase }}$ by scanning indiviual PVA/NBC phases using PFQNM with typical features to distinguish interphases between nanoparticles and polymer matrices based on the great variation of their nanomechanical properties. For instance, $W_{i \text { Interphase was }}$

17 detected by scanning along the $i$ th transverse plane for PVA/NBC phases. The same procedure was reapplied to determine $L_{j n t e r p h a s e}$ and $\mathrm{H}_{k \text { nterphase }}$ for the phase scanning among 19 the $j$ th longitudinal plane and $k$ th height plane, respectively (Refer to supporting information 20 for more details).

21 In this model, it has been assumed that uniform NBC dispersion takes place with two typical

22 categories of complete embedded and partially embedded NBCs within PVA matrices, as 23 illustrated in Figures 1(a) and (b), respectively. It is clearly demonstrated that the interphase 24 is surrounding between inner interface area and outer surface area bound by NBCs and PVA 25 matrices, respectively. By rearranging analytical equations according to Behmer and Hawkins 
1 [52] used for caculating the surface area of aninsotropic shape, we have derived the following

2 equations (1)-(4) for determining both outer surface area $\left(S A_{\text {outer Interface }}\right)$ and inner surface 3 area $\left(S A_{\text {inner Interface }}\right)$ of a wide range of fully and partially embedded particles. It is noted that 4 subscripts of 'f' and ' $p$ ' mean fully and partially embedded NBCs.

5

6

7

8

9

10

11

12

13 $\left(\text { SA } A_{\text {outer Interface }}\right)_{\mathrm{f}}$

$$
=a_{1}+b_{1} L_{\text {Interphase }}^{2}+c_{1} W_{\text {Interphase }}^{2}+d_{1} H_{\text {Interphase }}^{2}
$$

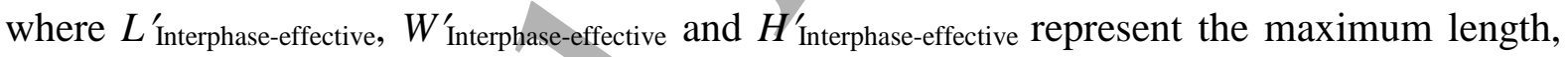

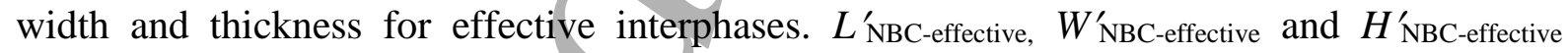
represent the maximum length, width and thickness for effective NBCs. the constants of $a_{1}$, $b_{1}, c_{1}$ and $d_{1}$ were determined to be $0.7439,0.3627,0.7006$, and 0.9979 , respectively, by fitting formula in equation (1) with the measurement of surface area (SA' ${ }^{\prime}$ outer Interface $)_{\mathrm{f}}[53,54]$ obtained from the AFM (See more details in supporting information).

After acquiring surface area data, the result were employed in detecting interphase/NBC volume $V_{\mathrm{NBC} / \text { Interphase }}$ and NBC volume $V_{\mathrm{NBC}}$ with respect to fully and partially embedded NBCs with modified equations being derived from Behmer and Hawkins [52] as below: 
1

2

3

4
$\left(S A_{\text {Outer Interface }}\right)_{\mathrm{f}}=e_{1} V_{\mathrm{NBC} / \text { Interphase }}^{f_{1}}$

\section{(8)}

5 The constants of $e_{1}, f_{1}$ were estimated to be 0.3824 and 0.3825 , respectively by fitting

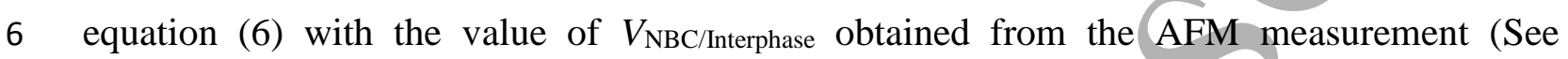

7 supporting information for more details).

8 Final step is to determine interphase volume $V_{\text {Interphase for fully or partially embedded NBCs }}$ 9 in PVA/NBC nanocomposites illustrated in Figures 1(a) and (b) as follows:

$$
\left(V_{\text {Interphase }}\right)_{\mathrm{f}}=V_{\mathrm{NBC} / \text { Interphase }}-V_{\mathrm{NBC}}
$$

$$
\left(V_{\text {Interphase }}\right)_{\mathrm{p}}=V_{\mathrm{NBC} / \text { Interphase effective }}-V_{\mathrm{NBC}} \text { effective }
$$

\section{Results}

\subsection{Modulus-gradient effect}

As illustrated in Figure 2(a), strong interactions between NBCs and PVA matrices appear to

17 be not only on NBC surfaces like other nanoparticles such as CNTs, nanosillcas, etc, but also

18 into entire NBC particles through the penetration of PVA molecules. Figures 2(b) and (c)

19 demonstrate a typical topographical image of PVA/NBC nanocomposite sample and the variation of elastic moduli from PVA matrices, interphases to NBCs, respectively. According to a cut line $A_{1} B_{1}$ shown in Figure 2(b), elastic modulus has significantly increased from the 22 lowest modulus level of $14.8 \mathrm{GPa}$ with respect to PVA matrices to the highest of $72.86 \mathrm{GPa}$ 23 for NBCs. In particular, the interphase exhibits a nearly linearly increasing relationship of its 
1 elastic modulus with scan distance except interphase boundary regions around PVA matrices

2 and NBCs alone, which is in range from 17.1 GPa near PVA region to 64.9 GPa around

$3 \quad$ NBCs with the measured interlayer thickness of $31.8 \mathrm{~nm}$. Average interphase modulus was

4 obtained from the solid black curve by discrete data averaging in Figure 2(d), which also

5 applied to average elastic moduli of NBCs and PVA matrices identified to be $78.4 \pm 4.9$ and

$6 \quad 24.25 \pm 4.2 \mathrm{GPa}$, respectively.

\section{$7 \quad$ 3.2. 3D interphase dimensions and modulus}

8 Elastic properties of interphases generally vary when surrounded by individual particles in

9 different sizes and shapes, thus leading to the change of interphase dimensions. Interphase

10 dimensions can be determined according to AFM height and adhesion images of PVA/NBC

11 composite samples, depicted in Figures 3(a) and (b), as well as 4(c) and (d), respectively. As

12 evidently seen in Figures 3(a) and (b), the height of NBCs is/much greater than that of PVA

13 with a linearly increasing tendency from interphase regions near PVA matrices to those close

14 to NBCs. NBCs can be easily distinguished from PVA matrices owing to their different

15 adhesion properties. It is clearly shown that the tip adhesion to PVA matrices of

16 nanocomposite sample is $10.76 \pm 3.42 \mathrm{nN}$, which is much greater than $2.1 \pm 0.87 \mathrm{nN}$ for

17 NBCs. Accordingly, interphase thickness is represented by the scan distances of 16 and 13

$18 \mathrm{~nm}$ with a sharp decreasing adhesion gradient from PVA matrices to NBCs on both sides of

19 selected material regions, as illustrated in Figure 3(d).

20 Interphase width $W_{\text {Interphase }}$, interphase length $L_{\text {Interphase }}$ and interphase height $H_{\text {Interphase }}$ was

21 measured among individual NBCs with associated results being presented in Figure 4(a). It

22 is evidently shown that the $L_{\text {Interphase, }} W_{\text {Interphase }}$ and $H_{\text {Interphase }}$ were measure to be 97,40 and 8

$23 \mathrm{~nm}$, respectively. As seen from Figures 4(b)-(e), despite the chaotic trend for interphase

24 thickness $\left(t_{\text {Interphase }}\right), H$ Interphase tends to decrease in a linear manner with an increase in $H_{\mathrm{NBC}}$ 
1 while $L_{\text {Interphase }}$ and $W_{\text {Interphase }}$ linearly increase with an increase in $L_{\mathrm{NBC}}$ and $W_{\mathrm{NBC}}$,

2 respectively.

3

4

5

A proposed mechanism model in terms of the effects of NBC dispersion and particlematrix interaction is demonstrated in Figures 5(a)-(e). It is identified in this study that there are two major NBC dispersion statuses within PVA matrices, namely uniform NBC dispersion and highly aggregated NBC dispersion in PVA/NBC nanocomposites. In the case of uniform NBC dispersion, two typical categories for the particle-matrix interaction can be addressed including completely embedded and partially embedded NBCs within PVA matrices, as shown in Figures 1(a) and (b), respectively. The former yields more effective interphases surrounding each side of NBCs (i.e. full coverage of NBCs by the interphases), which is considered as a good representation for effective load transfer from matrices to nanoparticles. The latter offers less pronouced interphase effectiveness than the former since some remarkable portions of NBCs are free of contact with PVA matrices, leading to the reduction of more effective interfacial regions. Interphase volume $V_{\text {Interphase }}$ is regarded as an essential characteristic parameter to evaluate the effects of fully or partially embedded NBCs in PVA/NBC nanocomposites, which can be specified as $\left(V_{\text {Interphase }}\right)_{\mathrm{f}}$ and $(V \text { Interphase })_{\mathrm{p}}$, respectively .

Figures 5 (c) displays the interphase modulus ( $\left.E_{\text {Interphase }}\right)$ as a function of $S A_{\text {outer interface }}$ and $S A_{\text {inner interface }}$ in the case of fully and partially embedded NBCs within PVA matrices in PVA/NBC nanocomposites, respectively. It is clearly revealed that with increasing $S A_{\text {outer }}$ interface and $S A_{\text {inner interface, }}, E_{\text {Interphase }}$ is improved remarkably in a non-linear manner accordingly. In particular, fully embedded NBCs yield consistently higher interphase modulus as opposed to partially embedded NBCs when either inner or outer interface is considered. 


\section{$1 \quad 3.3$ Particle debonding}

2 When NBC loading increased up to $5 \mathrm{wt} \%$, the interparticle spacing became smaller and

$3 \quad$ NBCs tended to be jointly connected with neighbouring particles side by side, as presented in

4 Figure 6(a), in which some sides of each particle became contact-free with PVA matrices.

5 Furthermore, a joint particle-to-particle formation may induce the phase separation leading

6 to the scattering of load transfer from PVA matrices to NBCs, as evidenced by the particle

7 overlap of NBCs in Figure 6(b).

8 At a high nanoparticle loading of $10 \mathrm{wt} \%$, NBC aggregation appeared to be mainefested

9 with a sign of large stacked-up NBC aggregates, depicted in Figure 6(c). As such, the

10 particle-to-particle separation occurred more evidently with resulting weak interfacial

11 bonding. Figure 6(d) reveals the modulus variation mapping on the surface cross-section of a

12 line scan (i.e. the line between two ovals from $\mathrm{A}_{4}$ to $\mathrm{B}_{4}$ ) shown in Figure 6(c), which

13 highlights two narrow zones with a sharp decline in elastic modulus.

\section{4. Discussion}

15 The interphase could be considered as transitional zones with a typical modulus gradient 16 from polymer matrices to reinforcements in order to achieve effective load transfer for the 17 improvement of composite mechanical properties. Our result reveals the existence of 18 interphase with excellent elastic properties in range from $25.32 \pm 3.4$ to $66.3 \pm 3.2 \mathrm{GPa}$. Owing to highly porous structures of NBCs, it makes them quite different from other carbon based nanofillers, thus facilitating the generation of highly positive capilary pressure to drive PVA 21 molecular chains into NBC pores, and further forming typical chemical bonding in nanocomposites [55, 56]. These NBC pores also possess 'mechanical anchoring' mechanism

$23[6]$ when interacting with PVA molecular chains, which means that a mechanical interlocking 24 phenomenon takes place due to the existence of PVA molecular chains inside BC surfaces 
1 [6]. Moreover, gradient mechanical properties at the interphase zone from NBCs to PVA

2 matrices could be associated with the number of hydrogeyen bonding from NBC surfaces to

3 polymer matrices, which plays an important role in controlling mechanical properties of

4 polymer nanocomposites [57, 58]. Nanoelastic behaviour of PVA demonstrates an elastic

5 modulus of $24.25 \pm 4.2 \mathrm{GPa}$ in this study, which is close to the value of 23.69 GPa previously

6 reported via the nanoidentation for PVA/chitsan (CS) copolymer coating [59]. However,

7 average elastic modulus of bulk PVA films was approximately $2.08 \mathrm{GPa}$ [36] at a

8 macroscopic level, which appeared to be much smaller in contrast with those of individial

9 amorphous and crystalline regions, obtained via PFQNM for PVA/NBC nanocomposites in

10 this study. Such a great difference in measurements can be ascribed to the following reasons:

11 (i) Though instrumental parameters have been calibrated prior to AFM measurements, the

12 shape function of the topmost probe tip may not be accurate enough for the low penetration

13 depth [24,25]. In this study, the low pentration force has been used, resulting in a low

14 penetration depth in order to minimise the effects of residual stresses as well as plastic

15 deformation from the neighbouring indent, with the provision of the high lateral resolution

16 capability particularly for nanointerphases. (ii) Nanosurface properties often vary from those

17 bulk properties because of the discrepancy in morphological structures between outer skins

18 on material surfaces and bulk films as well as the differences in nanomechanical behaviour

19 and bulk properties [24]. (iii) Despite the data reliability and repeatability of DMT modulus,

20 its determination using the PFQNM is apparently dintinct from tensile modulus for bulk

21 properties via conventional tensile testing owing to the use of different measurement

mechanisms [60].

23 The interphase identification bewteen PVA and NBCs are not limited to the variation of

24 nanoelastic behaviour, but could be distinguished based on the variation of height profiles

25 and adhesion properties, as shown in Figures 3(a)-(d). The adhesion of PVA matrices was 
1 found to be $10.76 \pm 3.42 \mathrm{nN}$, which appeared to be much greater than that of $\mathrm{NBCs}$ at

$22.1 \pm 0.87 \mathrm{nN}$. Such a finding was attributed to the hydrophilic nature of PVA with higher

3 adhension force when compared with hydrophobic NBCs. Furthermore, interphase thickness

4 was measured to be $13 \mathrm{~nm}$, as depicted in Figure 3(d), which was in good accordance with

5 the values of 13 and $12.5 \mathrm{~nm}$, previously determined for epoxy/graphene nanoplatelet (GNP)

6 composites and epoxy/graphene oxide (GO) nanocomposites, respectively [61].

7 Consequently, it is proven from our results that interphase thickness ( $\left.t_{\text {Interphase }}\right)$ is a non-

8 uniform and non-constant quantity as far as interphase dimensions are concerned. The

9 uniformity of interphase thickness ( $\left.t_{\text {Interphase }}\right)$ is most likely to be associated with the number

10 of chemical hydrogen bonds and physical roughness of NBC surfaces [4, 39, 62].

11 The major drawback in previous work lies in a simple assumption of one-dimensional

12 interphase layers with contant interphase thickness. Howeyer, our study demonstrated that

13 actual nanointerphases should be formed from a three-dimensional point of view to identify

14 the non-uniformity and dimensional variations of interphases in terms of $t_{\text {Interphase. }}$ Here we

15 successfully measured interphase dimensions, as shown in Figure 4(a). Moreover, It is cleraly

16 seen that there is no direct relation between $t_{\text {interphase }}$ and $t_{N B C}$, exhibited in Figure 4(b),

17 suggesting that interphase thickness may be independent of particle thickness.

18 Moreover, Figures 4 (c)-(e) indicate that interphase height tends to decrease in a linear

19 manner with an increase in NBC height while interphase length and interphase width linearly

20 increase with increasing NBC length and width, respectively. This phenomenon is ascribed to

21 more contact surface areas taking place between polymer matrices and nanoparticles in a

22 nanocomposite system [1]. Elastic properties of interphases are strongly affected by

23 interphase dimensions since interphase modulus tends to become higher with increasing outer

24 and inner interface surface area, as illustrated in Figures 5 (c). In particular, as for fully 25 embedded NBCs, a higher modulus increasing trend was evident as opposed to that based on 
1 partially embedded NBCs. This finding suggests that interphases can play a more important

2 role in enhancing mechanical properties of PVA/NBC nanocomposites with better bonded

3 matrix-filler interactions. Moreover, it has also been detected that the inner interface area

4 gives rise to relatively high overall interphase modulus in contrast with outer interface area.

5 As mentioned earlier by Liu et al. [61], the interphase zone with modulus gradient effect can

6 be divided into two different regions, namely Region 1 and Region 2 for a typical case in

7 Figure 5(d), in which Region 1 is in contact with NBC zones with relatively high interphase

8 density when compared with Region 2. Additionally, Fan et al.[63] reported that increasing

9 the interphase density inevitably led to the modulus increase for the same type of materials in

10 good accordance with high interphase modulus results obtained in case of inner interface

11 area. Apparently, the right boundary of Region 1 where inner interface is located is adjacent

12 to NBC zones with higher density and elastic modulus, as evidenced in Figure 5(d). The

13 relationship between interphase modulus and interphase volume is demonstrated in Figure

$145(\mathrm{e})$ on the basis of fully and partially embedded NBCs in PVA/NBC nanocomposites. It is

15 clearly seen that the significant enhancement of interphase modulus with increasing

16 interphase volume, which is similar to the case by increasing $S A_{\text {Interphase. }}$ The same applies to

17 fully embedded NBCs, resulting in much greater interphase modulus than partially embedded

counterparts in terms of interphase volume in PVA/NBC nanocomposites.

In addition to particle dimensions, particle dispersion and distribution patterns in polymer matrices are the other key factors in controlling interfacial bonding between NBCs and PVA matrices in PVA/NBC nanocomposites. When excessive amounts of NBCs are dispersed within PVA matrices, NBCs tend to agglomerate owing to their weak Van der Waals interactions. Additionally, increasing the particle loading inevitably decreases the

24 interparticle spacing, and thus hinders good particle dispersion [3]. Blighe et al.[64] reported 25 that the interparticle spacing was $6 \mathrm{~nm}$ in PVA nanocomposites reinforced with 10 vol\% 
1 single-walled carbon nanotubes (SWCNTs) with a clear sign of particle aggregation. As

2 such, the slipage phenomenon of stacked NBCs under mechanical loading gives rise to less

3 effective enhancement level for mechanical properties of nanocomposites. Moreover, Particle

4 agglomeration becomes manifested at high NBC contents depicted in Figures 6 (a) - (d). Such

5 phenomena can be explained by Li et al. [4] that in a nanocomposite system/nanoparticles

6 are brushed with modified layers of polymer matrices especially at a high particle volume

7 fraction. Additionally, 'modified polymer shells' surrounding different particles are

8 overlapped to form continuous phases, which means that such modified polymer shells

9 become the interphases with separated regions from 'parents' matrices', resulting in less

10 desirable properties. Furthermore, some NBCs are unable to actively interact with PVA

11 molecular chains leading to weaker interfacial bonding and higher possibility of phase

12 separation between NBCs and PVA matrices. This finding is indicative of the existence of

13 particle debonding effect between nanofillers and polymer matrices [15]. It is also worth

14 mentioning that lower interphase modulus can be associated with higher densities of

15 nanoparticles and polymer matrices as compared to that of interphase zones [52]. The

16 aforementioned NBC dispersion pattern in PVA/NBC nanocomposites suggests that there is a

17 percolation threshold for the NBC loading of $5 \mathrm{wt} \%$, beyond which nanomechanical

18 properties of interphases may diminish in good accordance with PVA/graphene oxide (GO)

19 nanocomposites $[63,64]$.

20 5. Conclusions

21 In this study, PFQNM was implemented to quantitatively characterise interphases of eco-

22 friendly PVA/NBC nanocomposites. This work demonstrates in detail a pioneering approach

23 for measuring 3D interphase dimensions as well as determining nanomechanical properties in

24 terms of interphase volume and surface area. Experimental characterisation results indicate

25 that interphase thickness appears to be non-uniform among individual particles and becomes 
1 independent of particle thickness. Moreover, it has been detected that interphase modulus in

2 PVA/NBC system is enhanced with increasing interphase volume. Nanomechanical

3 properties and sizes of interphases depend strongly on NBC dispersion status. Nevertheless,

4 increasing NBC loading was found to result in the decrease in intera-particle spacing leading

5 to particle agglomeration, causing the interfacial debonding effect between PVA matrices and

6 NBCs. This study demonstrates that two key geometric parameters of interphases surface

7 area and interphase volume are non-constant variables that can be influenced by NBC

8 dimensions and sizes, as well as NBC dispersion status. Our approach enlightens the

9 inclusion of actual interphase properties in theoretical and numerical modelling framework

10 rather than using a simple assumption of single interphase layer dimension and uniform

11 interphase properties in order to achieve more accurate prediction.

12 References

13 [1] Mousa M H, Dong Y and Davies, I J 2016 Inter. J. Polym. Mater. Polym. Biomater. 65 14225.

15 [2] Virgilio N, Favis B D, Pépin M F, Desjardins P and L'Espérance, G 2005 $16 \quad$ Macromolecules 382368.

17 [3] Jesson D A and Watts J F 2012 Polym. Rev. 52321.

18 [4] Li Y, Huang Y, Krentz T, Natarajan B, Neely T and Schadler L S 2016 19 Interface/Interphase in Polymer Nanocomposites Netravali A N, Mittal K L eds (Wiley, 20 Hoboken) 1.

21 [5] Drzal L T 1986 Epoxy Resins and Composites II Dušek K ed (Springer, Berlin) 1.

22 [6] Kim J K and Mai Y W 1998 Engineered Interfaces in Fiber Reinforced Composites 23 (Elsevier, Oxford).

24 [7] Lewis T J 2005 J. Phys. D-Appl. Phys. 38202.

25 [8] Gao S L and Mäder E 2002 Compos. Pt. A-Appl. Sci. Manuf. 33559. 
1 [9] Qu M, Deng F, Kalkhoran S M, Gouldstone A, Robisson A and Van Vliet K J 2011 Soft Matter 71066.

3 [10] Berriot J, Martin J F, Montes H, Monnerie L and Sotta P 2003 Polymer 441437.

4 [11] Litvinov V M, and Steeman P A M 1999 Macromolecules 328476.

5 [12] Pompe G and Mäder E 2000 Compos. Sci. Technol. 602159.

6 [13] Brown D, Marcadon V, Mélé P and Albérola, N D 2008 Macromolecules 411499.

7 [14] Li Y, Waas A M and Arruda E M 2011 J. Mech. Phys. Solids 5943.

8 [15] Gu Y, Li M, Wang J and Zhang Z 2010 Carbon 483229.

9 [16] Appiah K A, Wang Z L, Lackey W J 2000 Carbon 38831.

10 [17] Dwivedi H, Mathur R B, Dhami T L, Bahl O P, Monthioux M and Sharma S P 2006 Carbon 44699.

[18] VanLandingham M R, Dagastine R R, Eduljee RF, McCullough R L, Gillespie Jr. J W 1999 Compos. Pt. A-Appl. Sci. Manuf. 3075.

[19] Wang Y and Hahn T H 2007 Compos. Sci. Technol. 6792.

[20] Hodzic A, Kim J K, Lowe A E and Stachurski Z H 2004 Compos. Sci. Technol. 642185.

[21] Wright-Charlesworth D D, Peers W J, Miskioglu I and Loo L L 2005 J. Biomed. Mater. Res. Part A 74306.

[22] Gao S L, Mäder E and Zhandarov S F 2004 Carbon 42515.

[23] Kim J K, Sham M L and Wu J 2001 Compos. Pt. A-Appl. Sci. Manuf. 32607.

[24] Clifford CA and Seah M P 2005 Appl. Surf. Sci. 2521915.

[25]Yedla S B, Kalukanimuttam M, Winter R M and Khanna S K 2008 J. Eng. Mater. Technol. 130041010.

[26] Jafarzadeh S, Claesson, P M, Sundell P E and Pan J 2014, ACS Appl. Mater. Interfaces 6 19168. 
1 [27] Sababi M, Kettle J, Rautkoski H, Claesson P M and Thormann E 2012 ACS Appl. Mater.

2

3

4

5

6 [30] Amo C A and Garcia R 2016 ACS Nano 107117.

7 [31] Leja K and Lewandowicz G. 2010 Polish J. Environ. Stud. 19255.

8

9

10

11

12

13

14

15

16

17

18

19

20

21

22

23

24

25 Interfaces 45534. and Van Berkel G J 2015 ACS Nano 94260.

[29] Foster B 2012 Am. Lab. 4424.

[32] Huang D and Wang A 2013 RSC Adv. 31210. 17554.

[34] Pan Y S, Xiong D S, R Y 2007 Wear 2621021. Mater. 203322.

[36] Mousa MH and Dong Y 2017 ACS Sustainable Chem. Eng., 6467.

[37] Pakzad A, Simonsen J and Yassar R S 2012 Compos. Sci. Technol. 72314. Appl. Polym. Sci., 1283220. 214. Mater. Interfaces 812434.

[41] Zare Y. 2016. J. Appl. Polym. Sci. 13343824.

[42] Zare Y. 2016 Compos. Part A Appl. Sci. Manuf., 91, 132.

[43] Hu K, Gupta M K, Kulkarni D D and Tsukruk V V 2013 Adv. Mater. 252301.

[44] Pittenger B, Erina N and Su C 2009 Bruker Application Notes 128\# 1.

[28] Ovchinnikova O S, Tai T, Bocharova V, Okatan M B, Belianinov A, Kertesz V, Jesse S

[33] Yang X, Zhang X, Liu Z, Ma Y, Huang Y and Chen Y 2008 J. Phys. Chem. C 112

[35] Putz, K W, Compton, O C, Palmeri M J, Nguyen S T and Brinson L C 2010 Adv. Funct.

[38] Fortunati E, Puglia D, Monti M, Santulli C, Maniruzzaman M and Kenny J M 2013 J.

[39] Widjojo N, Chung T S, Weber M, Maletzko C and Warzelhan V 2011 J. Memb. Sci. 383

[40] Knauer K M, Jennings A R, Bristol A N, Iacono S T and Morgan S E 2016 ACS Appl. 
1 [45] Pittenger B, Erina N and Su C 2014 In Nanomechanical Analysis of High Performance Materials Tiwari A Ed. (Springer, Dordrecht) 31.

3 [46] van der Werf K O, Putman C A J, de Grooth B G and Greve J 1994 Appl. Phys. Lett. 65

4

5 1195.

[47] Voss A, Stark R W and Dietz C 2014 Macromolecules 475236.

[48] Derjaguin B V, Muller V M and Toporov Y P 1975 J. Colloid Interface Sci. 53314.

[49] Pittenger, B., Erina, N. and Su, C., 2012. Application Note\# 128

[50] Ohler B, 2007 Rev. Sci. Instrum. 78063701.

[51] Belikov, S., Alexander, J., Wall, C., Yermolenko, I., Magonov, S. and Malovichko, I., 2014, June. In American Control Conference (ACC), 1014). IEEE.

[52] Behmer D J and Hawkins C P 1986 Freshw. Biol. 16287.

[53] Erdoğan S T 2016 J. Mater. Civ. Eng. 2804016062.

[54] Parviz H, Cong C, Qiu C and Yu T 2013 Sci. Rep. 32593.

[55] Gray M, Johnson M G, Dragila M I and Kleber M 2014 Biomass Bioenerg. 61196.

[56] Brockhoff S R, Christians N E, Killorn R J, Horton R, Davis 2010 Agron. J. 1021627.

[57] Tashiro K and Tadokoro H 1981 Macromolecules 14781.

[58] Tashiro K and Kobayashi M.1991 Polymer 321516.

[59] Mishra S K and Kannan S 2014 J Mech Behav Biomed Mater 40314.

[60] Syed Asif S A, Wahl K J, Colton R J and Warren O L 2001 J. Appl. Phys. 901192.

[61] Liu Y, Hamon A L, Haghi-Ashtiani P, Reiss T, Fan B, He D and Bai J 2016 ACS Appl. Mater. Interfaces 834151.

[62] D'Souza N, Pendse S, Sahu L, Ranade A and Vidhate S 2016 In Interface/Interphase in Polymer Nahocomposites Netravali A N and, Mittal K L eds. (Wiley, Hoboken) 139.

[63] Fan H, Hartshorn C, Buchheit T, Tallant D, Assink R, Simpson R, Kissel D J, Lacks D J, Torquato S and Brinker C J 2007 Nat. Mater. 6418. 
1 [64] Zhao X, Zhang Q, Chen D and Lu P 2010 Macromolecules 432357.

2

3

4

5

6

7

8

9

10

11

12

13

14

15

16

17

18

19

20

21

22

23

24

25

26

26

27

28

29

30 


\section{$1 \quad$ List of Figures}

2 Figure 1. Schematic diagrams of (a) single fully embedded NBC and(b) single partially 3 embedded NBC with particle-matrix interactions.

4 Figure 2. (a) proposed scheme for PVA/NBC intercation, (b) 3D AFM modulus mapping 5 image of PVA/ $3 \mathrm{wt} \% \mathrm{NBC}$ nanocomposites, (c) modulus profile for sample was taken

6 along the red line $A_{1} B_{1}$, and (d) typical modulus data for 25 modulus profile samples along 725 different line scan region (LSR).

8 Figure 3. (a) 3D AFM height maps image of PVA/ 3 wt\% NBC nanocomposites, (b) height 9 maps profile of the sample was taken along the red line $A_{2} B_{2}$, (c) $3 D$ adheasion maps, and 10 (d) adhesion maps profile of sample was taken along the red line line $\mathrm{A}_{3} \mathrm{~B}_{3}$.

11 Figure 4. (a) maximum interphase dimensions along typical PVA/NBC interphases and relationships between NBC and interphase dimensions: (b) interphase thickness $\left(t_{\text {Interphase }}\right)$ and NBC thickness $\left(t_{N B C}\right),(\mathrm{c})$ interphase height $\left(H_{\text {Interphase-max }}\right)$ and NBC height $\left(H_{N B C}\right),(\mathrm{d})$ interphase length ( $\left.L_{\text {Interphase-max }}\right)$ and NBC length $\left(L_{N B C}\right)$ as well as (e) interphase width $\left(W_{\text {Interphase-max }}\right)$ and NBC width $\left(W_{N B C}\right)$.

Figure 5. AFM height mapping image of PVA/ NBC nanocomposites: (a) fully embedded NBCs and (b) partially embedded NBCs, (c) relationship between interphase area ( $\left.S A_{\text {Interphase }}\right)$ and interphase modulus ( $\left.E_{\text {Interphase }}\right)$, (d) schematic diagram for two proposed interphase zones and (e) relationship between interphase volume ( $\left.V_{\text {Interphase }}\right)$ and interphase modulus (Enterphase).

Figure 6. (a) Typical $3 \mathrm{D}$ AFM height mapping image of PVA $/ 5 \mathrm{wt} \% \mathrm{NBC}$ nanocomposite 22 surfaces with different effects: (a) joint edges of particles and (b) NBC overlapping, (c) 23 topographical image of PVA/10 wt\% NBC nanocomposite surfaces for phase separation and 24 (d) height mapping profile of nanocomposite sample cut along the red line $\mathrm{A}_{4} \mathrm{~B}_{4}$. 


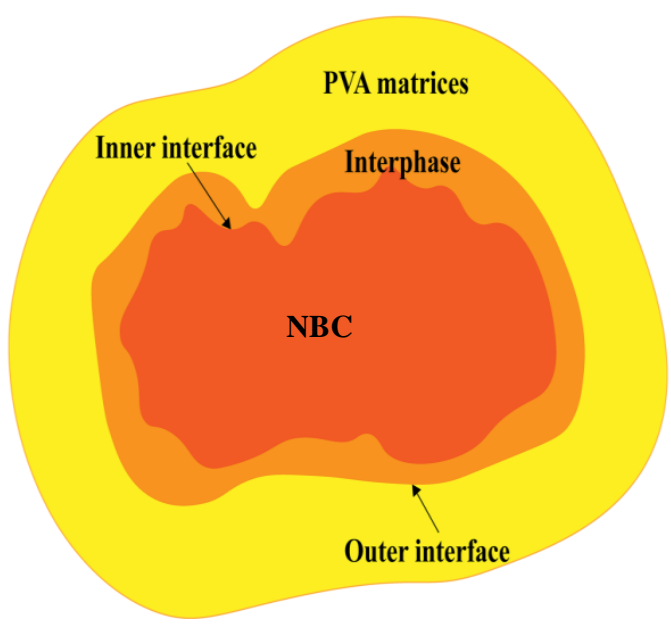

(a)

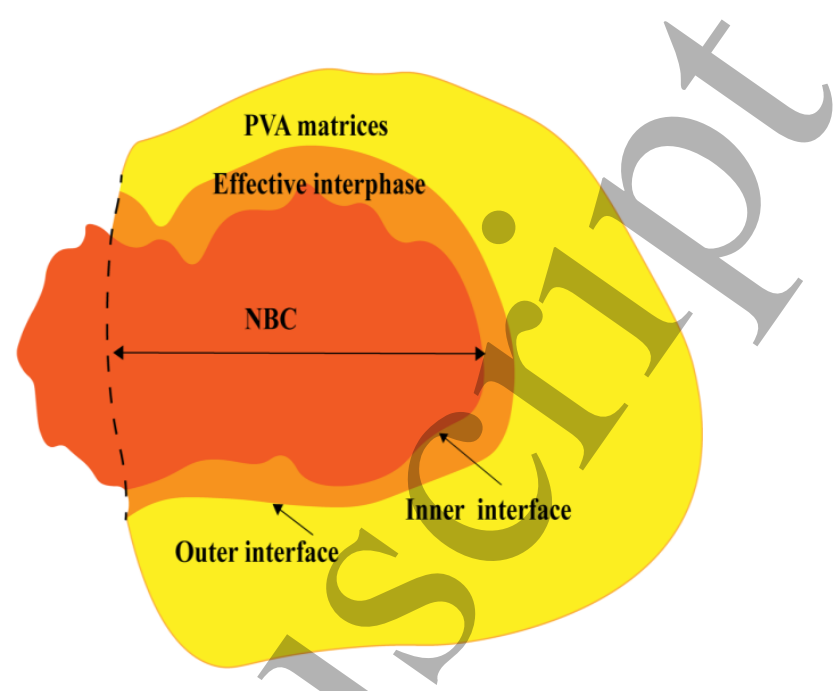

(b)

2

Figure 1 


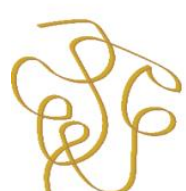
PVA chain
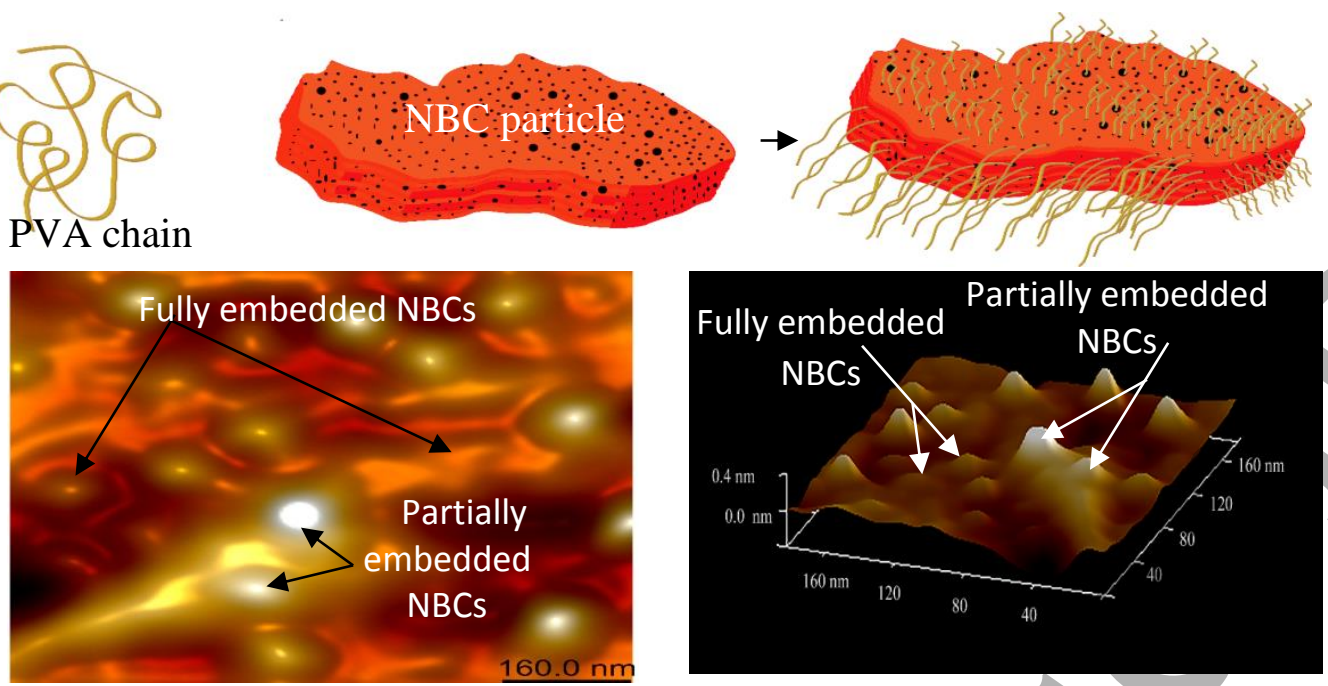

(a)

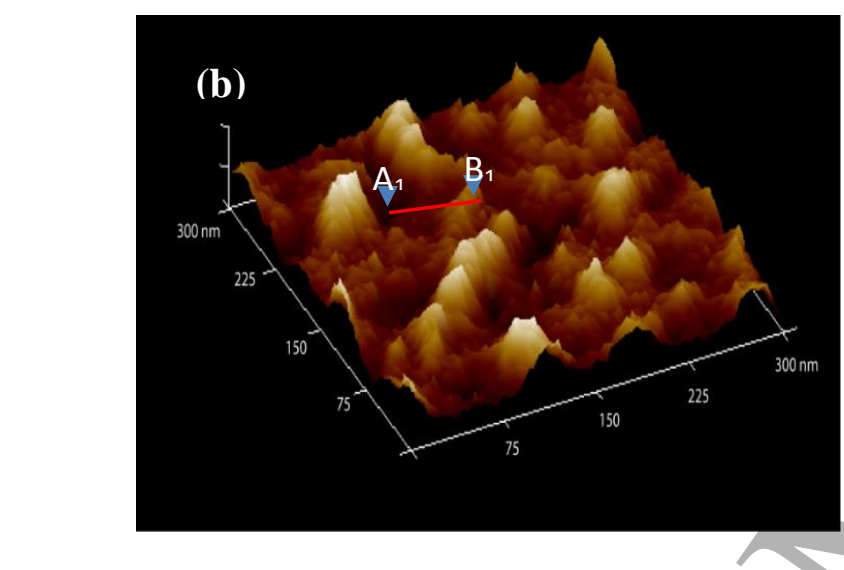

(a)
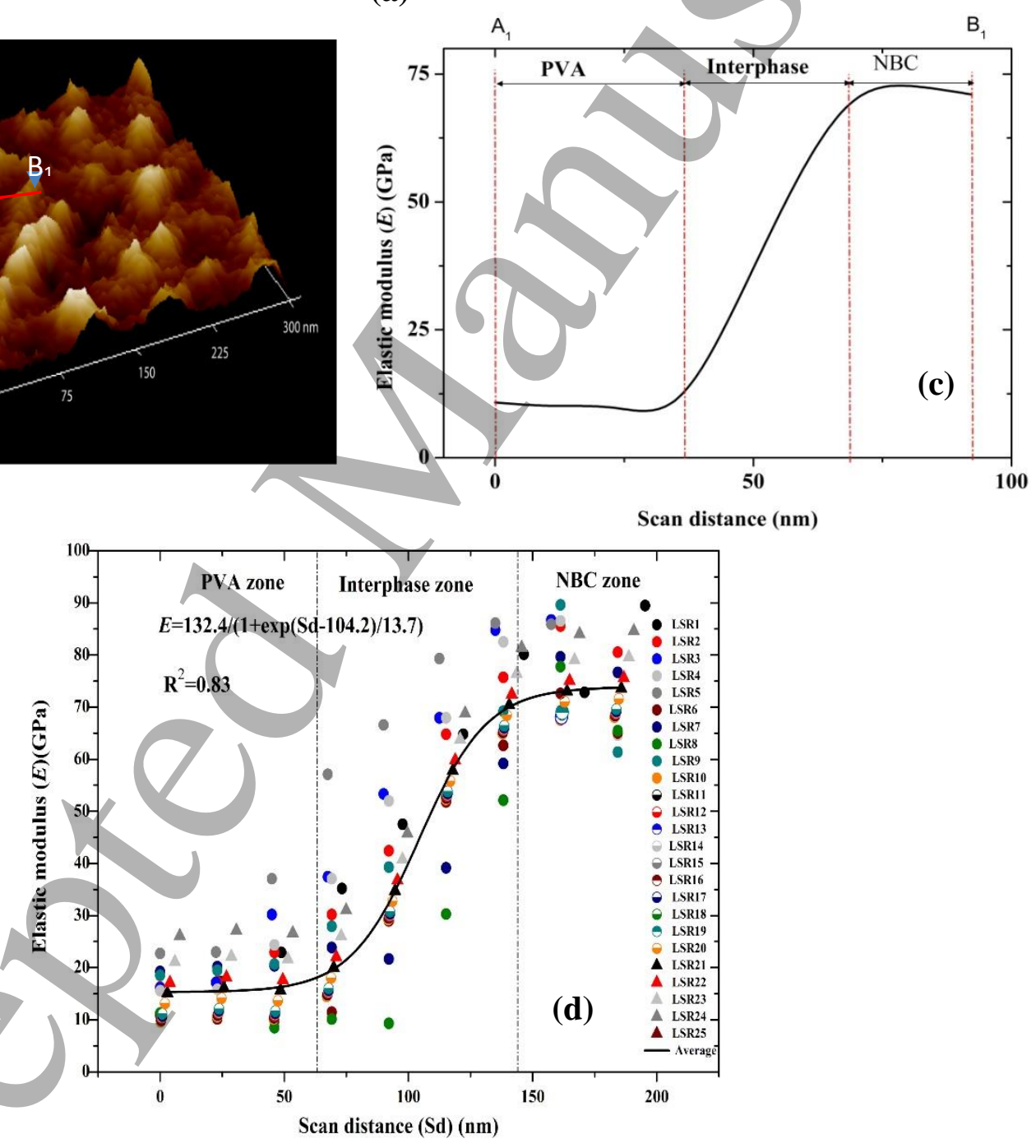

Figure 2 
1
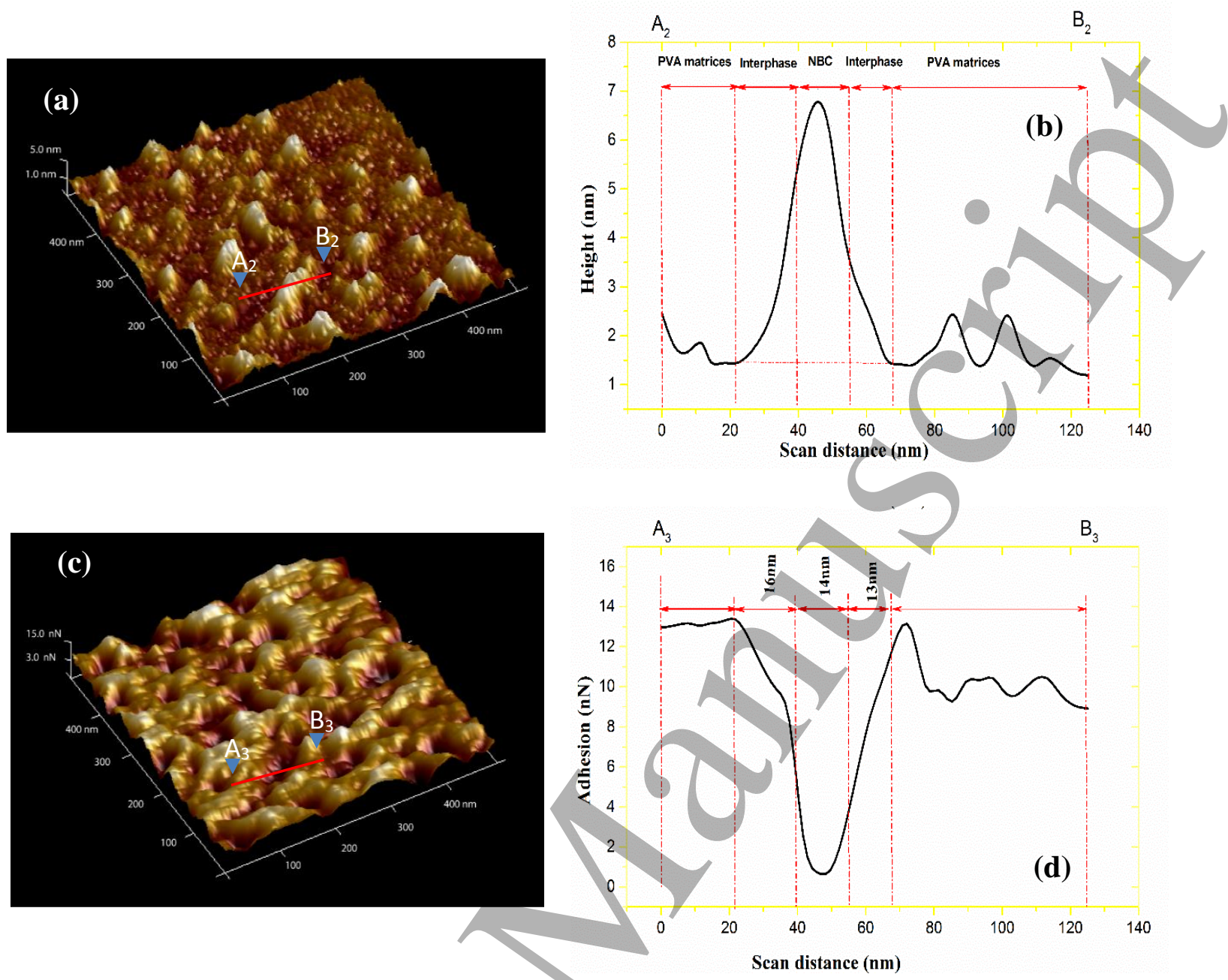

Figure 3

37
38

3

40

4

42


1

2
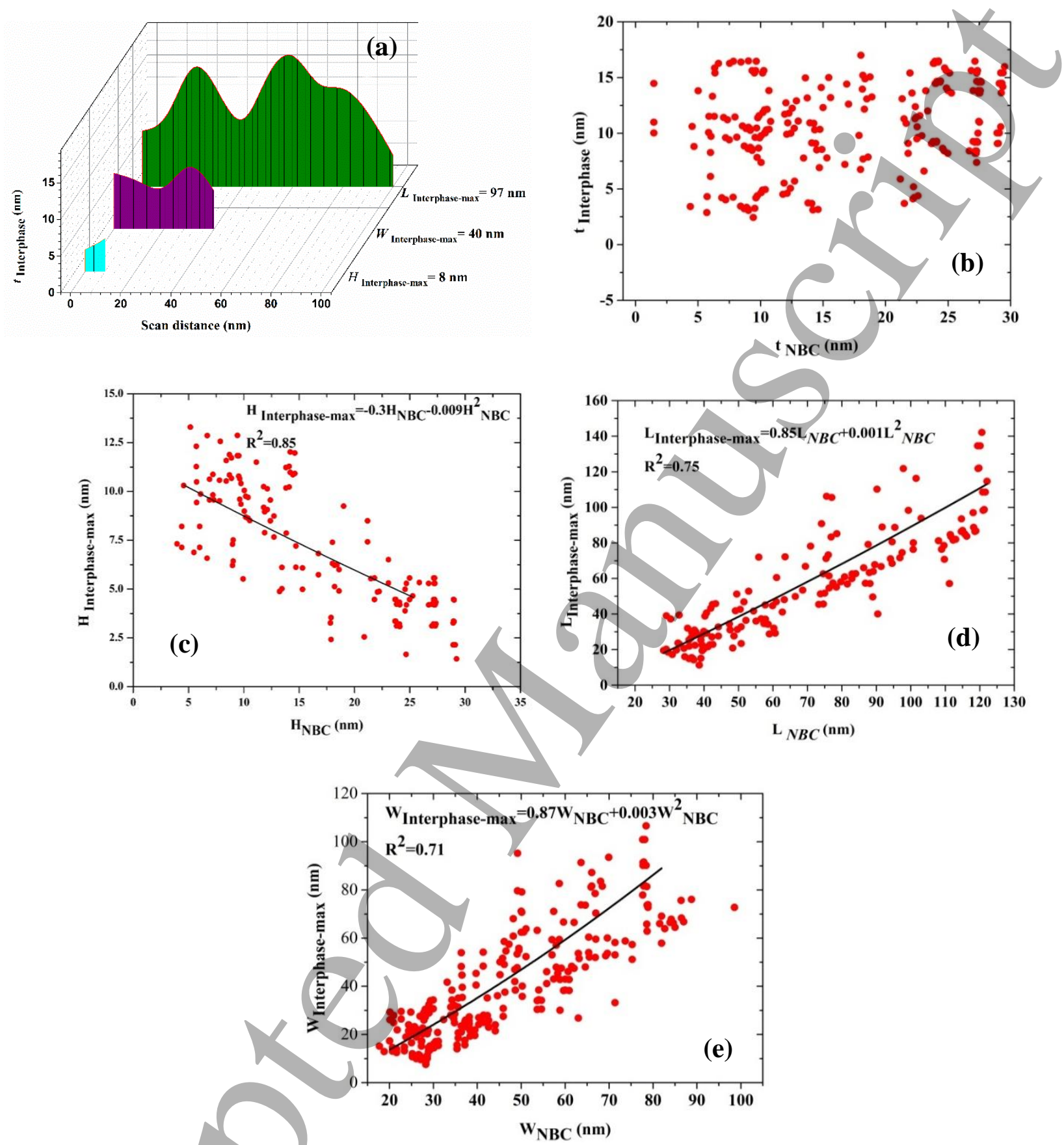

Figure 4 

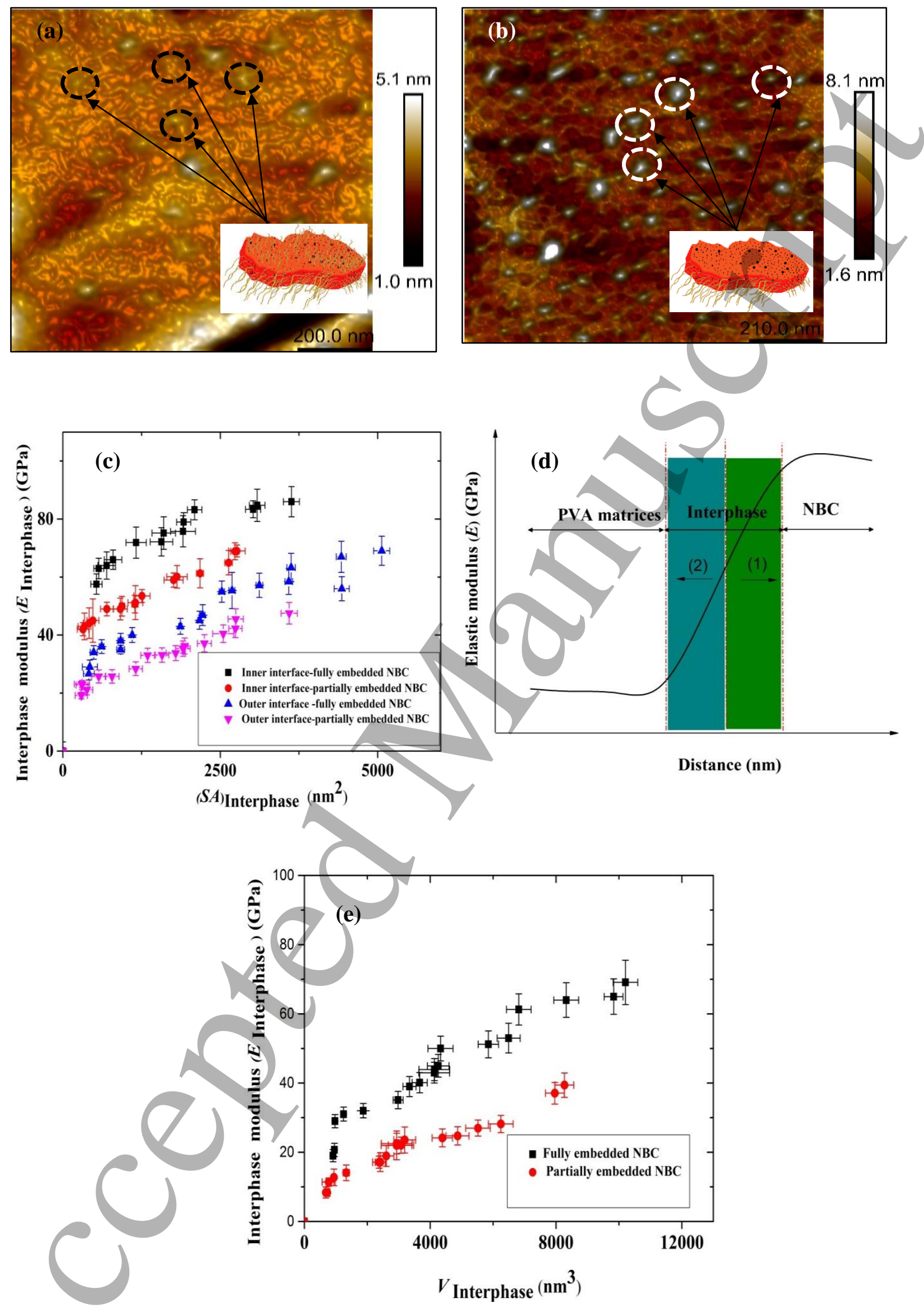

Figure 5 


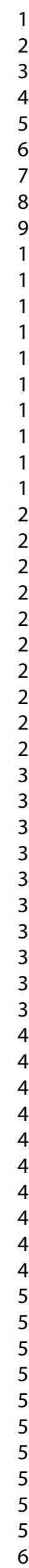
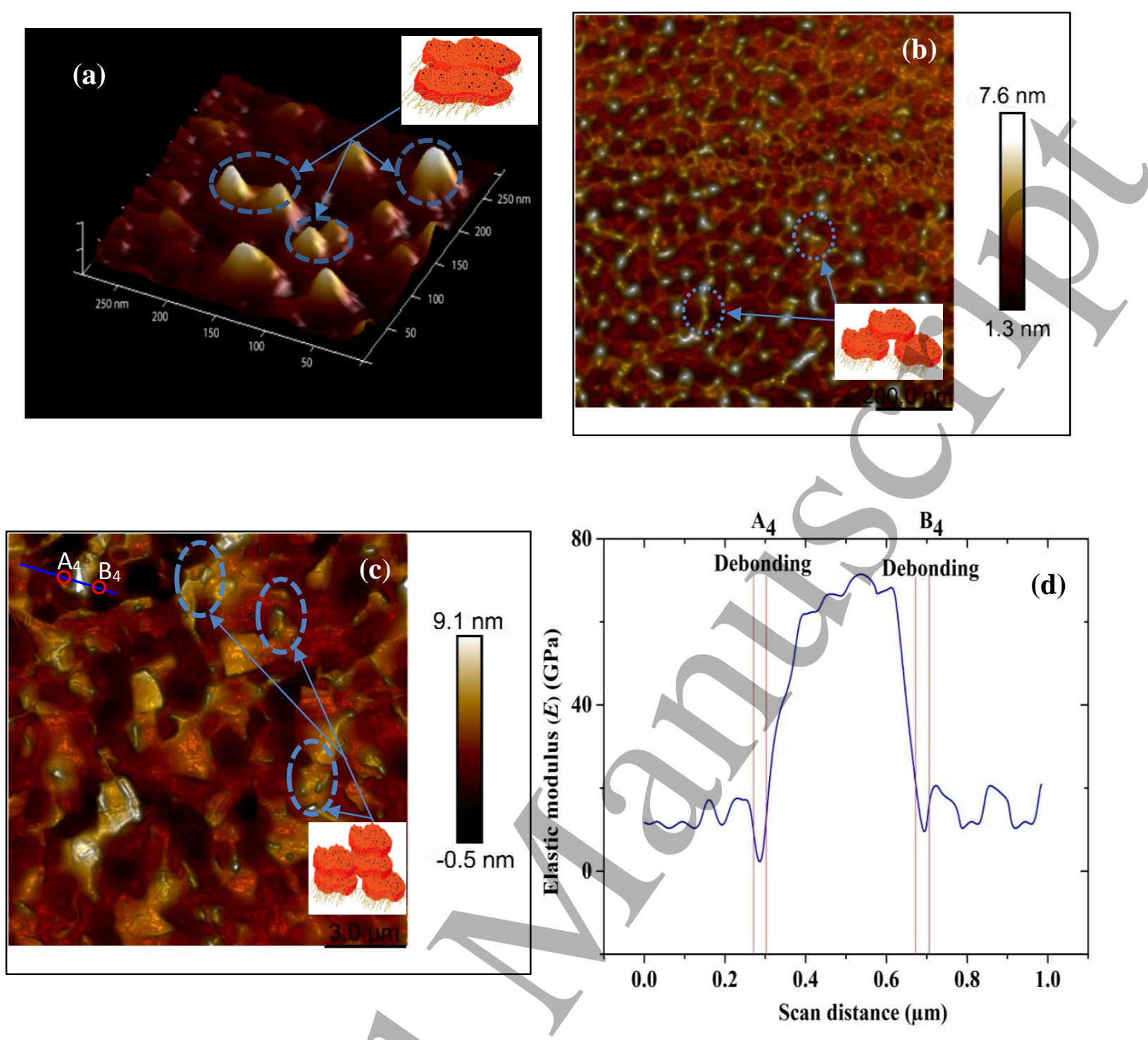

Figure 6 